Journal of Theoretical and Applied Mechanics, Sofia, Vol.49 No.1 (2019) pp. 94-104

INTERDISCIPLINARY TOPICS

\title{
AUTOMATIC BONE DRILLING IN HIP FRACTURES OSTEOSYNTHESIS
}

\author{
Tony Boiadjiev ${ }^{1}$, George Boiadjiev ${ }^{2}$, Kamen DelcheV ${ }^{3 *}$, \\ IVAN CHAVDAROV ${ }^{4}$, RUMEN KASTELOV ${ }^{5}$ \\ ${ }^{1}$ Institute of Information and Communication Technologies, Bulgarian \\ Academy of Sciences, Sofia, 1113, Bulgaria \\ ${ }^{2}$ Mathematics and Informatics, Sofia University, Sofia, 1164, Bulgaria \\ ${ }^{3}$ Institute of Mechanics, Bulgarian Academy of Sciences, Sofia, 1113, \\ Bulgaria \\ ${ }^{4}$ Institute of System Engineering and Robotics, Bulgarian Academy of \\ Sciences, Sofia, 1113, Bulgaria \\ ${ }^{5}$ Orthopaedic and Trauma Clinical Centre of Ministry of Interior, Sofia, 1606, \\ Bulgaria
}

[Received 11 October 2018. Accepted: 18 February 2019] doi: 10.7546/JTAM.49.19.01.09

\begin{abstract}
This paper presents an investigation of automatic bone drilling application in osteosynthesis of hip fractures. The real hip fractures osteosynthesis includes bone drilling from hip femur through the neck (along its axis) and the hip head to its far cortex avoiding joint penetration by hip head far cortex registration. The automatic execution of such a task by the Orthopedic bone Drilling Robot "ODRO” is the main contribution of this paper. A femoral head cortex registration algorithm is proposed for automatic drilling the proximal femur (hip joint). Experiments are provided and graphs illustrating the results are presented and commented to validate them.
\end{abstract}

KEY WORDS: orthopedic surgery, bone drilling, handheld robot.

\section{TERMINOLOGY AND NOTATION}

\section{Term/Symbol/Abbreviation Definitions}

Femur bone

Intracapsular hip fracture

Arthroplasty

Cortex
Bone in the human leg extending from the pelvis to the knee. Femoral head and neck fractures.

The surgical repair of a joint or the fashioning of a movable joint, using the patient's own tissue or an artificial replacement.

The hard exterior of bones.

\footnotetext{
*Corresponding author e-mail: kamen@imbm.bas.bg
} 
Osteosynthesis The process of mechanically bringing the ends of a fractured bone close together, as by wiring together or attaching to a metal plate.

ODRO Orthopedic bone drilling robot.

PI Proportional-integral control law.

Ids Integral component that gives information about the bone density deviation and is formed in a sliding time interval.

AU Arbitrary units of measurement of time - 1 unit is defined as the scoring time, i.e. the interval of time between two measurements.

\section{INTRODUCTION}

The robot application in the orthopedic surgery is a subject of various scientific researches since the last decades. The reason is the robots assure such a precision and accuracy of orthopedic manipulations, which cannot be achieved manually. Nowadays several robotized systems are used in the orthopedic surgery practice. ROBODOC and RIO (Robotic Arm Interactive Orthopedic System) are applied for HIP Arthroplasty and Knee Arthroplasty [1-3]. Renaissance system and Mazor X system are applied in spine surgery for spinal vertebrae $[1,4]$.

The large robot application in the orthopedic surgery is still difficult because of their high cost and the need of qualified assisting team, which must be trained to work with robots. The so-called robotized handheld systems are developed for automation of the bone drilling orthopedic manipulation. These systems can ensure high accuracy and precision of drilling operations and at the same time their costs are much lower, compared to the multifunctional orthopedic robots. Also their practical implementation is extensively simpler and they do not require procedures such as: preoperative planning based on computer tomography scan (CT scan), calibration, registration and intraoperative navigation. Examples for such systems are DRIBON [5,6], SMARTdrillR orthopedic handheld robotic drilling device [7, 8] and IntelliSense orthopedic surgical drill device $[9,10]$. The last one has an authorization for use in the orthopedic surgery in USA since 2015. The complexity in the execution of orthopedic surgeries differs. One significant group of such complicated manipulations represents surgeries, applied to hip-fractures. As next, it is important to categorize those in a crisp manner.

A hip-fracture is a break of upper quarter of the femur bone. There are three types of hip fractures: intracapsular, intertrochanteric and sub trochanteric ones [11]. They occur in spongy bone, which has rich blood supply and the fractures knit together no matter of the treatment method. The operative treatment gives better results together with the least time of patient recovery. Moreover, the recent years reveal the development and fast promotion of new biomechanical stress-resistant implants, which gives 
a wide choice of tools for inner fixation (screws, plates, pins) of unstable fractures. That helps to realize the best stability for any different type of fractures.

The fracture fixation stability depends on the bone quality, fracture geometry, reposition, choice and position of the implant. The first two factors are out of the surgeon control but the last three are on his responsibility.

Osteosynthesis is a surgical procedure, which stabilizes and joins the ends of fractured (broken) bones by mechanical devices such as metal plates, pins, rods, wires etc. For metal osteosynthesis of proximal femur fracture the implant moves through the side cortex and anchors into the hip head. The post-operative complications, which often occur at fracture treatment by osteosynthesis and require an implant change or arthroplasty, are the so called: hip head re-fracture and implant penetration into the joint capsule. The main reason for that is a wrong positioning of the screws into the femoral head-neck fragment. Aiming unification of the implant tip positioning, the hip head is separated to nine zones according to superior, inferior, anterior and posterior part of the head. Most authors prefer central or inferior position for placement of the screw into the hip head and strongly advise, that the anteriorsuperior location must be avoided [12]. As a criterion for implant position- the socalled Tip-to-Apex Distance index (TAD - index) is used. The optimal positioning corresponds to TAD - index minimization. For an instance, it is the best factor of prognosis to avoid the cut out of the hip head and when the TAD - index is less than $24 \mathrm{~mm}$, the cut outs are not registered [12].

Here must be mentioned, the robotized handheld systems are oriented to bi-cortical long bone drilling only. In this case the aim is to reach a minimal drill bit penetration after the far cortex and to give information for the drilling hole depth. According to the authors' knowledge: the reports, publications and results for application of robotized handheld systems in osteosynthesis of hip fractures are still not known.

The purpose of this work is to present an approach, how to realize maximally deep accurate drilling hole, with the clear aim to ensure the most stable fixation of the fractured hip neck bone by implants. The successful realization requires: a hip head cortex registration, during the automatic bone drilling; as well as an optimal implant positioning into the hip head, whereas the penetration into the joint is eliminated.

\section{Materials And Methods}

\section{SPECIMENS}

The experimental specimens are represented by femur bones of domestic pigs and acquired by a procurement process from licensed and state approved retail meat stores. The experimental setup is shown in Fig. 1. 


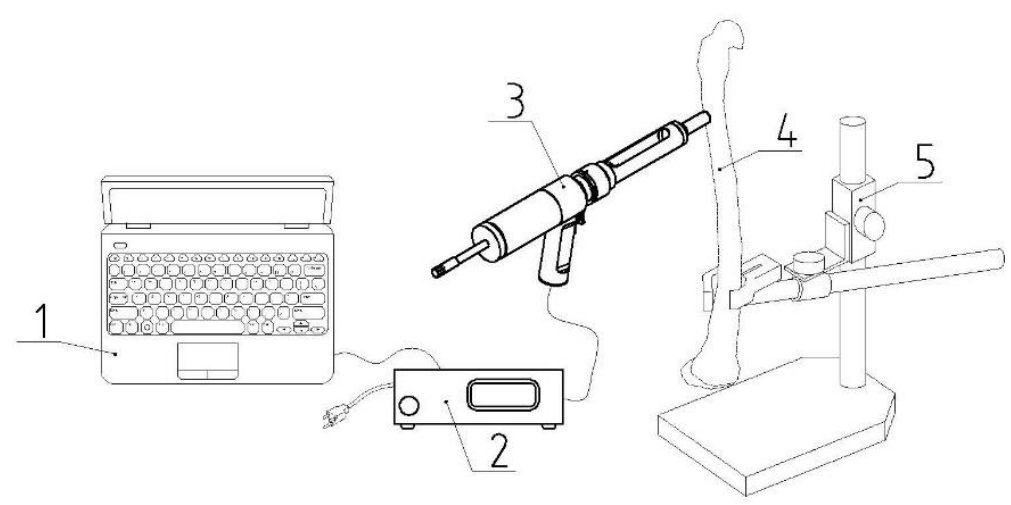

Fig. 1. Experimental setup: 1 - PC; 2 - control unit; 3 -drilling module; 4 - specimen (femur bone); 5 - vise.

\section{DRILLING SYSTEM}

The experiments are executed by the Orthopedic bone Drilling Robot "ODRO" (Fig. 2) [13-15]. The drilling starts at registration of the contact between the drill bit and the bone. The drilling process automatically stops when the corresponding manipulation criteria are fulfilled depending on the chosen working mode.

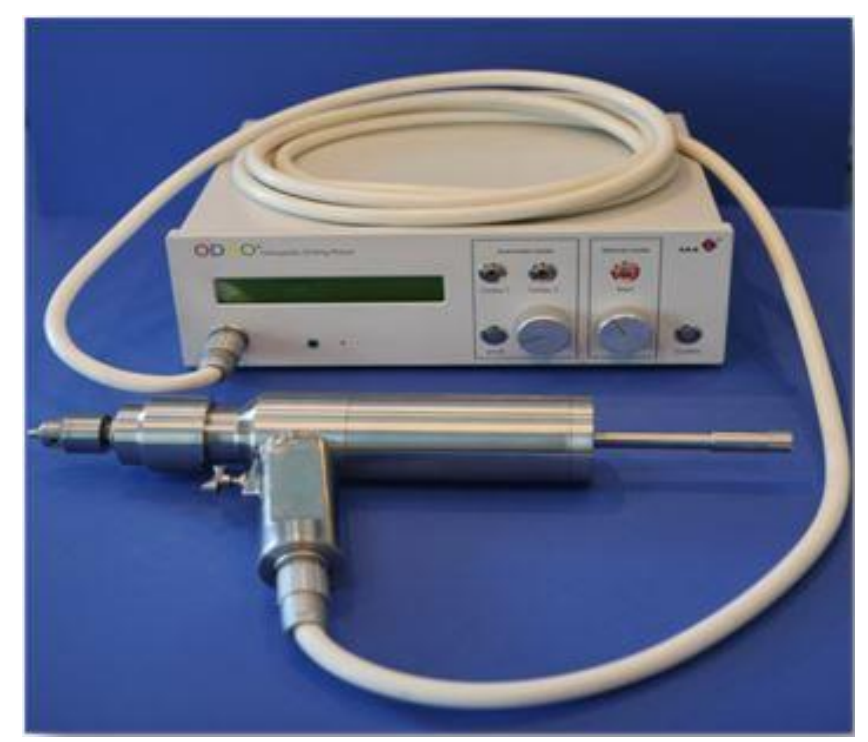

Fig. 2. (Color online) Orthopedic bone Drilling Robot "ODRO". 


\section{DATA ANALYSis}

A sensor inside the ODRO captures the thrust force signals. Specific sets of data are periodically sent to the PC for future analysis. The deviation of the thrust force in time is graphically presented (in Microsoft Excel) and analyzed. Time is expressed in arbitrary units (AU) of measurement; 1 unit is defined as the scoring time, i.e. the interval of time between two measurements.

\section{RESUlTS}

The control of the feed motion (translation) is realized by a thrust force feedback. modified proportional-integral (PI) control law is used to calculate the new position, i.e. number of steps of the linear drive stepper motor (respectively the drill-bit position) which must be reached in a given interval of time [16].

The hip head cortex registration depends on the bone density evaluation in the current drilling zone. Thus, an integral component Ids is formed in a sliding window in the same current drilling zone. It gives information about the bone density deviation [16]. Decreasing of Ids indicates drilling in an area of higher bone density and its increasing, vice versa, i.e. drilling in an area of lower bone density. On the base of this component the algorithm for far cortex registration of the hip head is realized. The latter leads to the following conclusions:

- during the hip head drilling along the neck the resistant force increases for the sake of going through the intersection area of principal tensile group and principal compressive group [17]. This intersection forms so called cross section area where the hip head has the most density and there Ids $<0$;

- after the end of cross section area the resistant force decreases (Ids $>0$ ) and arises again close to the hip head cortex (Ids $<0$ ).

Such a deviation of the thrust force depends on the drilling area. If the drilling is executed closely to the neck wall medial or lateral way the thrust force stays relatively constant with negligible arising in cross section area. Moreover, for patients having osteoporosis the last effect is negligible all the time. As the bone features are not known before the drilling then an additional criterion must be involved to determine the reason for higher bone density: the registration of the "cross section" area or the far cortex.

Based on the experiments in [16] an evaluation of the distance between the cross section area end and the head cortex beginning was done. It varies in the range of 6-12 $\mathrm{mm}$ in dependence on the hip head size. Taking into account the proximal femur size (from lateral cortex to the hip head end) it can be determined (with enough accuracy) that the distance mentioned above corresponds to $10 \%$ of the whole size 
of proximal femur. Such an evaluation allows (with the help of already determined proximal femur size by the X-ray image) taking synonymy decision for hip head cortex registration. The proximal femur size is set as a parameter for the working mode choice as it is shown in Fig. 3.

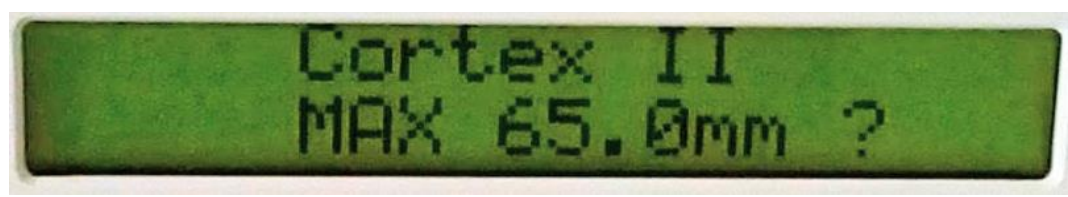

Fig. 3. (Color online) Setting of the proximal femur size in the drilling area.

For the particular case the bone size is $65 \mathrm{~mm}$. The setting of this parameter means: if the thrust force increases during the drilling (Ids $<0$ ) in a distance less than $58.8 \mathrm{~mm}$ from the contact point with the bone, then this result corresponds to registration of the beginning of cross section area. If the thrust force increases in a distance greater than $58.8 \mathrm{~mm}$ from the contact point with the bone, that corresponds to hip head cortex registration.

The setting of working mode for proximal femur drilling and hip head cortex seeking is shown in Fig. 4.

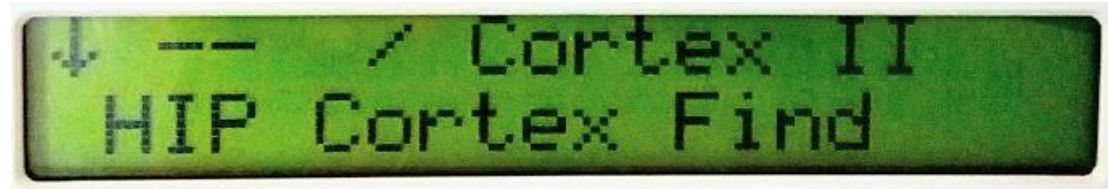

Fig. 4. (Color online) Setting of working mode for proximal femur drilling and hip head cortex seeking.

The experimentally obtained results in this work for far cortex registration during proximal femur drilling along the neck axis are presented in Fig. 5. The measured proximal femur length is $65 \mathrm{~mm}$. The hip head cortex registration is in a distance $61.7 \mathrm{~mm}$ from the contact point.

After the contact with the bone (at $100 \mathrm{AU}$ ) the drilling is executed with feed speed $1 \mathrm{~mm} / \mathrm{s}$ (from 100 to $150 \mathrm{AU}$ ). In this interval of time the thrust force in the drilling area is identified. After that the drilling goes on with feed speed of $4 \mathrm{~mm} / \mathrm{s}$. The drilling of the lateral cortex is finished at $233 \mathrm{AU}$ and the drilling automatically stops. After pressing the start button again the drilling starts at 480 AU. From 480 to $550 \mathrm{AU}$ the drilling is executed with feed speed of $2 \mathrm{~mm} / \mathrm{s}$. The thrust force in the drilling area of spongy zone of the bone is identified in this interval of time. After that the drilling proceeds with feed speed $4 \mathrm{~mm} / \mathrm{s}$. The beginning of the "cross section" 
Thrust Force Fact; Integral com ponent Ids

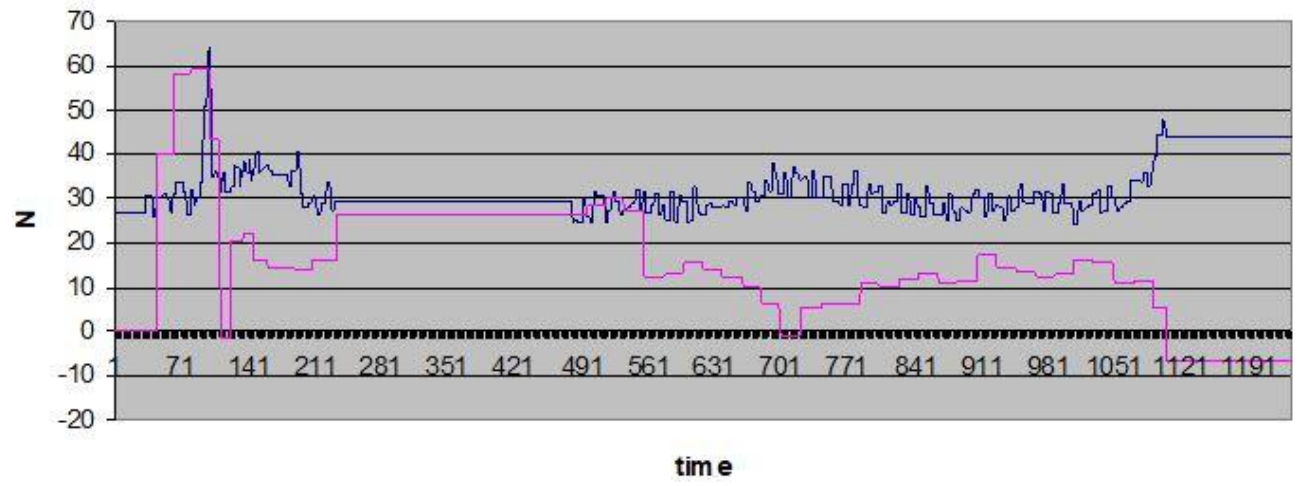

Fig. 5. (Color online) Far cortex registration during pig proximal femur bone drilling along the neck axis. $V_{\max }=4 \mathrm{~mm} / \mathrm{s}$; Drill Bit $2.8 \mathrm{~mm}$; Total time $24.84 \mathrm{~s}, 1235$ AU; Hole depth $61.7 \mathrm{~mm}$ (preliminary set depthMAX $=65.0 \mathrm{~mm}$ ). The values of the Integral component Ids (red line) are scaled by multiplication of $10^{-1}$.

area is at $700 \mathrm{AU}$ where Ids $<0$. The hip head cortex registration occurs at $1107 \mathrm{AU}$ where Ids again takes values less than zero. Then the drilling automatically stops and

\section{Thrust force Fact; Integral component Ids}

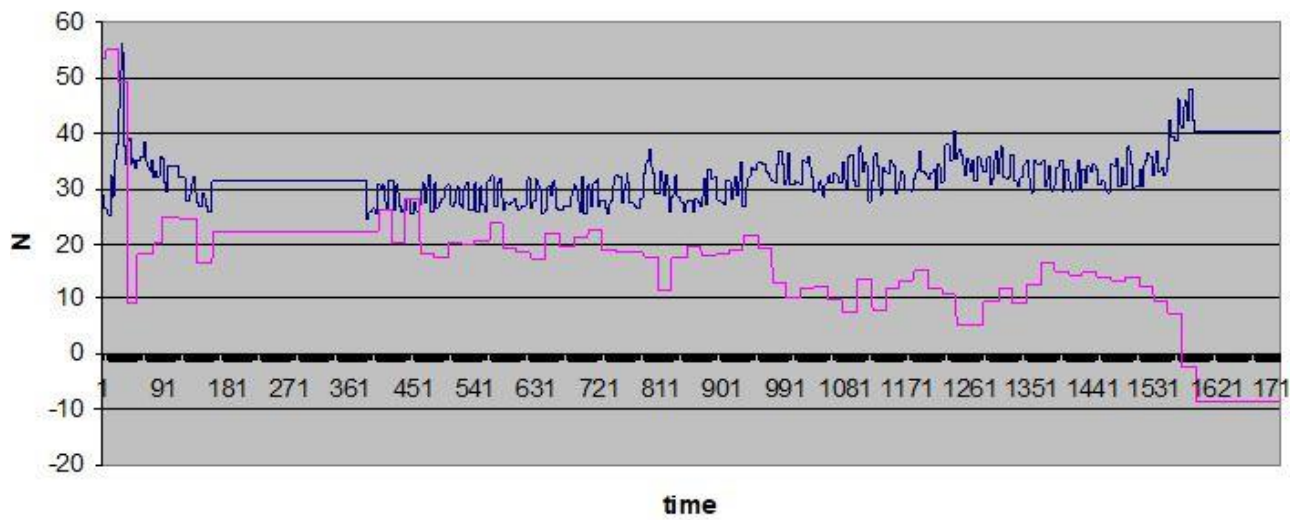

Fig. 6. (Color online) Far cortex registration during pig proximal femur bone drilling closely to the neck wall laterally. $V_{\max }=2 \mathrm{~mm} / \mathrm{s}$; Drill Bit $2.8 \mathrm{~mm}$; Total time $35.031 \mathrm{~s}, 1712 \mathrm{AU}$; Hole depth $60.5 \mathrm{~mm}$ (preliminary set depth MAX mm $=64.0 \mathrm{~mm}$ ). The values of the Integral component Ids (red line) are scaled by multiplication of $10^{-1}$. 
the robot takes out the drill bit at the initial position.

The results, which are obtained for a far cortex registration - during proximal femur drilling closely to the neck wall laterally; are presented in Fig. 6.

The measured proximal femur length, during drilling along the neck axis, is $64 \mathrm{~mm}$. The hip head cortex registration is in a distance $60.75 \mathrm{~mm}$ from the contact point.

The contact with the bone is realized at $23 \mathrm{AU}$. The lateral cortex drilling is finished at $160 \mathrm{AU}$. The hip head cortex registration is at $1590 \mathrm{AU}$ where Ids $<0$. At that moment the drilling automatically stops and the robot takes out the drill bit at the initial position.

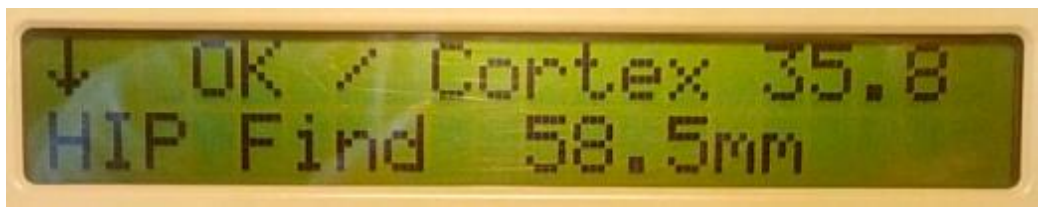

Fig. 7. (Color online) Information for the final result of the manipulation.

When the whole drilling process is completed the final result appears on the display (Fig. 7). For this particular case the information, which is represented on the display means:

- "HIP Find" - successful hip head cortex registration;

- $58.5 \mathrm{~mm}$ - the distance from the contact point of the drill bit with the bone to the beginning of the hip head cortex

- "OK" - no missing steps of the stepper motor are registered during the drilling, i.e. the distance of $58.5 \mathrm{~mm}$ corresponds to the depth of the realized hole with accuracy $0.1 \mathrm{~mm}$

- $35.8 \mathrm{~N}$ - value of the reference force related to identified force [16].

A check for a hip head end is running, as a synchronized concurrent process during the execution of the far cortex registration algorithm. In the case of an automatic drilling stop, related to the hip head end registration, the drill bit penetration in the hip head cortex is in the range of $0-1 \mathrm{~mm}$, which is not dangerous for implant penetration into the joint [16].

A preliminary set value of the hole depth (parameter "MAX - mm") is used for determination of the zone, where the decision for hip head end registration is taken. But it has another very important role from a manipulation safety viewpoint. When 
this parameter is set (in our case $65 \mathrm{~mm}$ ) a hole deeper than the preliminary set value cannot be drilled automatically.

To summarize, in this working mode the drilling automatically stops, if one of the following three conditions is fulfilled:

- hip head cortex registration;

- hip head end registration;

- reaching the preliminary set depth.

This condition (criterion), which triggers the drilling to automatically stop, is represented as an information output on the display after the end of the drilling process.

\section{Discussion}

The drilling through the lateral cortex along the hip neck axis, which goes as much as possible close to the far cortex hip head, is important especially for specific manipulation in osteosynthesis of hip fractures. Such a manipulation, which purpose is stable implant fixation, can be successfully executed only manually under continuous X-ray control. Please note: the deeper the drilled hole, the stable fixation is realized. On the other hand the robot assistance in orthopedic surgery significantly improves many surgical manipulations. This exemplifies another reason for the recent growth of the handheld robotized systems development and application in the scientific research and industrial practice. As it was noted in introduction, they are developed for bicortical long bone drilling, explicitly aiming to reach a minimal drill bit penetration after the end of far cortex drilling. During the application process, the author's team conducts a wide range of experiments not only for automatic bi-cortical long bone drilling, but also for femoral hip head structure. In [15] the experimental specimens, related to the femoral hip drilling, were represented by human hip heads, surgically removed from patients, during hip joint arthroplasty at a state hospital. Please note: such situation does not occur at real osteosynthesis and is helpful explicitly for experiments. In reality the hip fractures osteosynthesis includes the drilling in the whole area - from the hip femur, through the neck (along its axis) to the hip head.

Please note: the automatic execution of such drilling processes represents better results in general, therefore the successful solution of such a complex surgical task is the main contribution of this paper.

\section{CONCLUSIONS}

The algorithm for hip head far cortex registration by an automatic bone drilling, which is proposed in this work: allows executing this manipulation without $\mathrm{X}$-ray 
control and guarantees deepest hole without penetration in the joint. This contributes successfully to the realization to achieve the most stable implant fixation. After the manipulation process is finished, the information for the depth of the realized drilling hole is presented on the display with $0.1 \mathrm{~mm}$ accuracy. This procedure allows a precise choice of the implant size and its optimal positioning in the hip head.

The application of the algorithm, proposed here, and its realization by an automatic bone drilling execution support successfully also the application of minimally invasive surgery techniques in hip fractures osteosynthesis, as well as reduction of the post-operative period of the patient recovery.

\section{REFERENCES}

[1] M. Hoeckelmann, I.J. Rudas, P. Fiorini, F. Kirchner, T. Haidegger (2015) Current Capabilities and Development Potential in Surgical Robotics. International Journal of Advanced Robotic Systems; DOI: 10.5772/60133.

[2] R.A. BeASLE (2012) Medical Robots: Current System and Research Directions. Journal of Robotics; DOI: 10.1155/2012/401613.

[3] N. Sugano (2013) Computer-Assisted Orthopaedic Surgery and Robotic Surgery in Total Hip Arthroplasty. Clinics in Orthopedic Surgery 5 19; DOI: 10/4055/cios.2013.5.1.1.

[4] www.mazorrobotics.com

[5] M. Louredo, I. DiaZ, J.J. Gil (2012) DRIBON: A Mechatronic Bone Drilling Tool. Journal of Mechatronics 22 1060-1066.

[6] F. Accini, I. DiaZ, J.J. Gil (2016) Using an Admittance Algorithm for Bone Drilling Procedure. Computer Methods and Programs in Biomedicine 123 150-158; DOI: $10.1016 / j . c m p b .2015 .10 .003$.

[7] http://www.smartmeddevices.com/

[8] http://www.smartmeddevices.com/smartdrill/

[9] http://www.mcginleyorthopaedicinnovations.com/index.php?/pages/drill

[10] https://www.medgadget.com/2015/03/intellisense-bone-drill-with-auto-depthmeasurement-and-edge-detection-fda-cleared-video.html

[11] M. Butler, M. Forte, R.L. Kane, S. Joglekar, S.J. Duval, M. SWIONTKOWSKI T. WILT (2009) Treatment of Common Hip Fractures. Evidence Report/Technology Assessment No. 184, AHRQ Publication No. 09-E013. Rockville, MD. Agency for Healthcare Research and Quality; available at https://www.ahrq.gov/downloads/pub/evidence/pdf/hipfracture/hipfracture.pdf.

[12] M.R. Baumgaertner, S.L. Curtin, D.M. Lindskog, J.M. Keggi (1995) The Value of the Tip-Apex Distance in Predicting Failure of Fixation of Peritrochanteric Fractures of the Hip. Journal of Bone and Joint Surgery-American Volume 77 10581064 . 
[13] G. Boiadjiev, R. Kastelov, T. Boiadjiev, V. Kotev, K. Delchev, K. ZaGURSKI, V. VITKOV (2013) Design and Performance Study of an Orthopaedic Surgery Robotized Module for Automatic Bone Drilling. International Journal of Medical Robotics and Computer Assisted Surgery 9 455-463; DOI: 10.1002/rcs.1479.

[14] G. Boiadjiev, R. Kastelov, T. Boiadjiev, K. Delchev, K. Zagurski (2016) Automatic Bone Drilling - More Precise, Reliable and Safe Manipulation in the Orthopaedic Surgery. Journal of Theoretical and Applied Mechanics 46 51-64.

[15] T. Boiadjev, G. Boiadjiev, K. Delchev, K. Zagurski, R. Kastelov (2017) Far Cortex Automatic Detection Aimed FOR Partial or Full Bone Drilling by a Robot System in Orthopaedic Surgery. Biotechnology \& Biotechnological Equipment 31 200205; DOI: 10.1080/13102818.2016.1234947.

[16] T. Boiadjiev, R. Kastelov, G. Boiadjiev, K. Delchev, K. Zagurski (2017) Automatic Bone Drilling by Femoral Head Structure Detection. Biotechnology \& Biotechnological Equipment; DOI: 10.1080/13102818.2017.1407256.

[17] J.Y. Kwon, H. Naito, T. Matsumoto, M. TanaKa (2012) Osteocyte ApoptosisInduced Bone Resorption in Mechanical Remodeling Simulation - Computational Model for Trabecular Bone Structure. In: "Apoptosis and Medicine" Chapter 2; available at DOI: $10.5772 / 50301$ 